\title{
Adaptação climática no contexto das cidades brasileiras: reflexões à luz da Agenda 2030 para o Desenvolvimento Sustentável
}

\begin{abstract}
Resumo
Partimos aqui da hipótese que os Objetivos do Desenvolvimento Sustentável (ODS), propostos pela Agenda 2030 da Organização das Nações Unidas (ONU), podem se configurar como mecanismos importantes para redesenhar a agenda governamental e os modelos de gestão das cidades brasileiras nos próximos anos. Nosso objetivo, com este artigo, é discutir de que forma as cidades brasileiras têm se beneficiado (ou não) da Agenda 2030 e de seus ODS (em especial, 07, 11 e 13) como uma estratégia de promoção de sustentabilidade. A metodologia deste trabalho segue as orientações de uma abordagem de natureza qualitativa, na qual se utilizam das pesquisas bibliográfica e documental como instrumentos de coleta de dados para alcance de seus resultados. Nossos resultados permitem concluir que, no contexto do Brasil, os desafios de efetivação da capacidade urbana às mudanças climáticas alinhadas à Agenda 2030 demandam mais comprometimento de todos os setores da sociedade em virtude das assimetrias urbanas e sociais das cidades. A criação de mecanismos que possibilitem a implementação da Agenda 2030 é imprescindível devido ao histórico de negligência das questões ambientais nas agendas dos governos municipais do país.
\end{abstract}

Palavras-chave: Brasil. Capacidade adaptativa. Mudanças climáticas. Planejamento urbano. Sustentabilidade.

\section{Para citar este artigo:}

TEIXEIRA, Rylanneive Leonardo Pontes; PESSOA, Zoraide Souza; ARAÚJO, Ana Célia Baía; DIAS, Eric Mateus Soares. Adaptação climática no contexto das cidades brasileiras: reflexões à luz da Agenda 2030 para o Desenvolvimento Sustentável. PerCursos, Florianópolis, v. 21, n.46, p. 05 - 24, maio/ago. 2020.

\section{Rylanneive Leonardo Pontes}

Teixeira

Doutorando em Estudos Urbanos e Regionais pela Univ. Federal do

Rio Grande do Norte - UFRN. Brasil

pontesrylanneive@gmail.com

\section{Zoraide Souza Pessoa}

Doutora em Ambiente e

Sociedade pela Universidade

Estadual de Campinas -

UNICAMP. Professora do

Programa de Pós-Graduação em Estudos Urbanos e Regionais da Univ. Federal do Rio Grande do Norte - UFRN.

Brasil

zoraidesp@gmail.com

\author{
Ana Célia Baía Araújo \\ Mestra em Estudos Urbanos e \\ Regionais pela Univ. Federal do \\ Rio Grande do Norte - UFRN. \\ Brasil \\ araujo.acba@gmail.com
}

\section{Eric Mateus Soares Dias}

Mestrando em Estudos Urbanos

e Regionais pela Univ. Federal do

Rio Grande do Norte - UFRN.

Brasil

ericmateusemsd@gmail.com 


\title{
Climate adaptation in the context of Brazilian cities: reflections to light of the 2030 Agenda for Sustainable Development
}

\begin{abstract}
We start here with the hypothesis that the Sustainable Development Goals (SDGs), proposed by the 2030 Agenda of the United Nations (UN), can be configured as important mechanisms to redesign the governmental agenda and the management models of Brazilian cities in the coming years. In this sense, with this article, we aim to discuss how Brazilian cities have benefited (or not) from Agenda 2030 and their SDGs (in particular, 7, 11, and 13) as a strategy to promote sustainability. To this end, this work's methodology follows the guidelines of a qualitative approach, in which bibliographic and documentary research is used as data collection instruments to achieve its results. The results obtained with this article allow us to conclude that, in the Brazilian context, the challenges of realizing urban capacity to climate change aligned with the 2030 Agenda demand more commitment from all sectors of society due to urban and social asymmetries of cities. In the Brazilian case, creating mechanisms that allow the 2030 Agenda implementation is essential to the extent that we experience a historical second place for environmental issues in the municipal government agendas in the country.
\end{abstract}

Keywords: Brazil. Adaptive Capacity. Climate changes. Urban planning. Sustainability. 


\section{Introdução}

Um dos grandes desafios para as próximas décadas é o de efetivar a capacidade de gestão das cidades que tenha como estratégia a adaptação climática, essencial para os modelos de planejamento urbano numa perspectiva sustentável. Em certa medida, esse desafio requer alterações nos modelos atuais de planejamento urbano, que incorporam de forma superficial a dimensão ambiental no contexto do desenvolvimento urbano ao longo do tempo.

No contexto brasileiro, as dimensões territoriais do país conformam um conjunto complexo de cidades, em que as dinâmicas de crescimento urbano nem sempre são acompanhadas por um planejamento que oriente seus caminhos. As assimetrias entre as cidades brasileiras são muitas, organizadas em um arranjo de cidades cujas estruturas populacionais e de desenvolvimento social, econômico e ambiental são muito diferenciadas e que dependem dos modelos de gestão urbana e governamental que as caracterizam, o que gera um cenário de grandes vulnerabilidades, desigualdades, incertezas e, consequentemente, de riscos. Esse cenário é preocupante, tendo em vista que os principais problemas das cidades não foram resolvidos e tendem a avolumar-se nas próximas décadas, gerando cenários de incertezas, especialmente nas grandes cidades e metrópoles (PESSOA, 2012).

Esse cenário está associado aos muitos problemas das cidades brasileiras e do mundo, como a falta de acesso à moradia acessível e adequada, com saneamento básico integrado a sistemas de gestão eficiente dos resíduos sólidos, e redes de abastecimento e tratamento de efluentes que primem pelo reaproveitamento dos sistemas hídricos que as abastecem. Poderíamos nos estender e falar dos inúmeros problemas das cidades brasileiras, mas não é o propósito deste artigo.

Na presente análise, partimos da hipótese que os Objetivos do Desenvolvimento Sustentável (ODS), propostos pela Agenda 2030 da Organização das Nações Unidas (ONU), podem se configurar como mecanismos importantes para redesenhar a agenda governamental e os modelos de gestão das cidades brasileiras nos próximos anos. 0 nosso objetivo é discutir de que forma as cidades brasileiras têm se beneficiado (ou não) 
da Agenda 2030 e de seus ODS (em especial, 07, 11 e 13) como uma estratégia de promoção de sustentabilidade. Para tanto, a metodologia deste trabalho segue as orientações de uma abordagem de natureza qualitativa, fazendo uso das pesquisas bibliográfica e documental enquanto instrumentos de coleta de dados. A pesquisa bibliográfica consistiu no levantamento de referencial teórico-conceitual sobre planejamento urbano, mudanças climáticas e capacidade adaptativa urbana; enquanto a pesquisa documental levou em consideração a análise dos documentos referentes à Agenda 2030 e seus ODS (particularmente 07, 11 e 13) e o Plano Nacional de Adaptação à Mudança do Clima (PNA).

Para embasar essa discussão, além deste momento introdutório, temos outras cinco seções. Na primeira, apresentamos o planejamento urbano enquanto um instrumento importante para o enfrentamento dos riscos e efeitos impostos localmente pelas mudanças climáticas. Na segunda, mensuramos o quanto as cidades do Nordeste do Brasil são sustentáveis (ou insustentáveis). Na terceira, discutimos sobre as energias renováveis como uma alternativa para solucionar ou pelo menos atenuar os problemas agravados pelas mudanças climáticas. Na quarta, nos debruçamos sobre a análise da adaptação climática no contexto das cidades brasileiras a partir da análise do PNA e da Agenda 2030 e de seus ODS. Para finalizar, na quinta e última, trazemos as principais considerações finais sobre o desafio de efetivação da capacidade adaptativa às mudanças climáticas no contexto brasileiro.

\section{Planejamento urbano como gestão preventiva dos riscos e impactos das} mudanças climáticas nas cidades brasileiras

O ordenamento territorial é compreendido por Moraes (2005) e Almeida (2007) como um instrumento de organização e planejamento do território que tem por objetivo organizar a ocupação e o uso do solo, e orientar a gestão territorial e de riscos que está recebendo incidência humana. Nesses termos, entendemos que: 
O ordenamento territorial constitui a expressão territorial das políticas econômica, social, cultural e ecológica. Nesse sentido, seu entendimento caminha na direção de um "disciplinamento" no uso do território, de modo a compatibilizar, ou, ao menos, diminuir eventuais conflitos existentes nas diversas ações públicas e privadas que alteram dinamicamente os conteúdos físicos, sociais, econômicos e culturais contidos no território. (BRASIL, 2005, p. 63)

No contexto brasileiro, a política de ordenamento territorial tem vínculo com as políticas de desenvolvimento urbano e regional devido à extensão territorial do país e à necessidade de desconcentração populacional e de riqueza, mas também com as políticas de departamentos setoriais e da macroeconomia que têm efeitos sobre os territórios das cidades (RÜCKERT, 2007). Dentro dessa política maior, temos o planejamento urbano que, no contexto das mudanças climáticas, exerce papel central no enfrentamento dos efeitos desse fenômeno, porque possibilita a indução de transformações sustentáveis nesses sistemas, permitindo a inserção de novas atitudes e práticas de ocupação e uso do solo urbano, que alteram os estilos de vida das populações que vivem nesses espaços, auxiliando no enfrentamento das mudanças climáticas (APOLLARO; ALVIM, 2017).

Nessa perspectiva, ainda que o planejamento urbano funcione como meio crucial de avançar no enfrentamento das mudanças climáticas, especialmente em termos de adaptação climática1, cabe destacar que os planos diretores municipais do Brasil, enquanto principais instrumentos de planejamento urbano local, não estão preocupados com a diminuição das emissões de Gases de Efeito Estufa (GEE), tampouco com a adaptação aos efeitos das mudanças climáticas. Esse aspecto pode ser corroborado em estudos recentes sobre planejamento urbano e mudanças climáticas, como os realizados por Sathler, Paiva e Baptista (2019) e Espíndola e Ribeiro (2020).

Sobre isso, Di Giulio et al. (2019a) salientam que a ausência de ações de prevenção e resposta aos efeitos das mudanças climáticas no âmbito dos planos diretores municipais dificulta avançar no processo de adaptação climática, na medida em que compromete a oportunidade de as cidades estarem atrelando seu plano diretor aos

\footnotetext{
1 Entendemos a adaptação climática como um conjunto de ajustamentos realizado nas cidades, a fim de antecipar os efeitos das mudanças climáticas e, assim, reduzir a vulnerabilidade aos riscos dessas mudanças (IPCC, 2007).
} 
processos de adaptação climática, perdendo, consequentemente, a oportunidade de melhorar sua capacidade adaptativa às mudanças climáticas. Para Eakin, Lemos e Nelson (2014), essa capacidade consiste no potencial que as cidades têm em buscar por respostas de adaptação climática, de maneira que assimilem os efeitos dessas mudanças.

Reconhecemos, então, que as políticas públicas ou ações de enfrentamento das mudanças climáticas em associação e articulação com as estratégias de planejamento urbano numa perspectiva de gestão preventiva dos riscos são essenciais e cada vez mais importantes para a redução das condições de vulnerabilidade e possíveis situações de riscos, com consequências para o desenvolvimento humano, social e econômico integrado aos princípios da sustentabilidade ambiental.

Assim sendo, o planejamento urbano em associação com as estratégias de enfrentamento das mudanças climáticas contribui para o fortalecimento do ODS 13 ("ação contra a mudança global do clima”), visto que buscam por reduzir a pobreza e/ou as condições de vulnerabilidade (de jovens, de comunidades marginalizadas, entre outros) ou construir e/ou reforçar as cidades com uma infraestrutura resiliente. Nesse ínterim, é importante mensurar as ações que podem conduzir as cidades e que interferem, em certa medida, nos modelos de desenvolvimento regional, conforme refletiremos no tópico em sequência.

\section{Mensuração de cidades sustentáveis no Nordeste do Brasil através de indicadores}

O desenvolvimento regional envolve questões regionais de natureza social, econômica, ambiental etc., inclusive problemas que decorrem do desenvolvimento urbano e que findam extrapolando a escala territorial das cidades. O desenvolvimento regional considera um panorama de políticas e dinâmicas que se relacionam a um conjunto de cidades, com similaridades entre si. Na região Nordeste do Brasil, o desenvolvimento regional é afetado por questões históricas e políticas que estão presentes em todo o desenvolvimento urbano da região, resultando em cidades e regiões 
metropolitanas construídas através das desigualdades em seus territórios (ARAÚJO, 2019).

Mensurar o quanto cada uma das dimensões urbanas do desenvolvimento regional precisa da atenção da gestão e do planejamento de políticas públicas torna-se mais complexo quando se observam governos ainda arraigados nos interesses e conflitos por poder nas regiões, especialmente do Nordeste. Assim, analisamos indicadores da proposta de Cidades Sustentáveis (CS) da ONU, idealizada como alternativa para se repensar um desenvolvimento urbano e regional mais sustentável, através do ODS 11, "Cidades e comunidades sustentáveis". De acordo coma Plataforma ODS online do Instituto Brasileiro de Geografia e Estatística (IBGE, 2018), os indicadores oficiais já disponíveis para o Brasil referentes ao ODS 11 são os apresentados no Quadro 1.

Quadro 1 - Indicadores referentes às metas do ODS 11

\begin{tabular}{|c|c|}
\hline Indicadores & Metas a que se referem \\
\hline $\begin{array}{l}\text { A) Proporção de população urbana } \\
\text { vivendo em assentamentos } \\
\text { precários, assentamentos informais } \\
\text { ou domicílios inadequados }\end{array}$ & $\begin{array}{l}11.1 \text { - Até } 2030 \text {, garantir o acesso de todos à } \\
\text { habitação segura, adequada e a preço acessível, } \\
\text { e aos serviços básicos e urbanizar as favelas }\end{array}$ \\
\hline $\begin{array}{l}\text { B) Número de mortes, pessoas } \\
\text { desaparecidas e pessoas } \\
\text { diretamente afetadas atribuído a } \\
\text { desastres por } 100 \text { mil habitantes }\end{array}$ & $\begin{array}{l}11.5 \text { - Até } 2030 \text {, reduzir significativamente o } \\
\text { número de mortes e o número de pessoas } \\
\text { afetadas por catástrofes e substancialmente } \\
\text { diminuir as perdas econômicas diretas causadas } \\
\text { por elas em relação ao produto interno bruto } \\
\text { global, incluindo os desastres relacionados à } \\
\text { água, com o foco em proteger os pobres e as } \\
\text { pessoas em situação de vulnerabilidade }\end{array}$ \\
\hline $\begin{array}{l}\text { C) Proporção de governos locais que } \\
\text { adotam e implementam estratégias } \\
\text { locais de redução de risco de } \\
\text { desastres em linha com as } \\
\text { estratégias nacionais de redução de } \\
\text { risco de desastres }\end{array}$ & $\begin{array}{l}\text { 11.b - Até 2020, aumentar substancialmente o } \\
\text { número de cidades e assentamentos humanos } \\
\text { adotando e implementando políticas e planos } \\
\text { integrados para a inclusão, a eficiência dos } \\
\text { recursos, mitigação e adaptação às mudanças } \\
\text { climáticas, a resiliência a desastres; e } \\
\text { desenvolver e implementar, de acordo com o } \\
\text { Marco de Sendai para a Redução do Risco de } \\
\text { Desastres 2015-2030, o gerenciamento holístico } \\
\text { do risco de desastres em todos os níveis }\end{array}$ \\
\hline
\end{tabular}

Fonte: Elaboração própria a partir de IBGE (2018). 
Os indicadores do Quadro 1 mostram uma relação entre as metas do ODS e as pautas de gestão de riscos urbanos, desastres e adaptação, e habitação e resiliência urbana. Considerando esses três indicadores ( $A, B$ e C) com dados referentes ao Brasil e aos estados da Bahia (BA), do Ceará (CE), de Pernambuco (PE) e do Rio Grande do Norte (RN) como recorte do Nordeste, devido as suas expoentes importâncias no contexto regional, obtivemos os resultados conforme apresentados no Quadro 2.

Quadro 2 - Resultados de indicadores do ODS 11 por estados do Nordeste, Brasil (2018)

\begin{tabular}{|c|c|c|c|c|c|c|}
\hline Indicadores & Ano & BR & RN & CE & PE & BA \\
\hline $\begin{array}{l}\text { A) Proporção de população urbana } \\
\text { vivendo em assentamentos } \\
\text { precários, assentamentos informais } \\
\text { ou domicílios inadequados }\end{array}$ & 2010 & & $54,7 \%$ & $59,3 \%$ & $54,0 \%$ & $51,0 \%$ \\
\hline $\begin{array}{l}\text { B) Número de mortes, pessoas } \\
\text { desaparecidas e pessoas } \\
\text { diretamente afetadas atribuído a } \\
\text { desastres por } 100 \text { mil habitantes }\end{array}$ & $\begin{array}{l}2015 \\
2016 \\
2017\end{array}$ & & $\begin{array}{l}145,3 \\
136,6 \\
57,0\end{array}$ & $\begin{array}{l}397,6 \\
309,7 \\
316,3\end{array}$ & $\begin{array}{l}275,7 \\
173,7 \\
864,6\end{array}$ & $\begin{array}{l}316,5 \\
354,7 \\
308,0\end{array}$ \\
\hline $\begin{array}{l}\text { C) Proporção de governos locais } \\
\text { que adotam e implementam } \\
\text { estratégias locais de redução de } \\
\text { risco de desastres em linha com as } \\
\text { estratégias nacionais de redução } \\
\text { de risco de desastres }\end{array}$ & $\begin{array}{l}2010 \\
2011 \\
2012 \\
2013 \\
2014 \\
2015\end{array}$ & $\begin{array}{l}41,6 \% \\
42,2 \% \\
42,8 \% \\
43,2 \% \\
43,5 \% \\
44,6 \%\end{array}$ & & & & \\
\hline
\end{tabular}

Fonte: Elaboração própria a partir de IBGE (2018).

A partir da análise do Quadro 2, observamos níveis médio-altos de pessoas afetadas por desastres, pessoas vivendo em condições de moradia urbana inadequadas, bem como governos ainda pouco mobilizados na construção de estratégias de combate às condições de vulnerabilidades e situações de riscos urbanos. Nesse sentido, apreendemos que ainda há muito para o Brasil evoluir em termos de políticas de desenvolvimento regional que contemplem medidas de um desenvolvimento urbano sustentável, inclusivo, adaptativo e resiliente. 
O desenvolvimento regional tem sofrido impacto com 0 volume de empreendimentos de energias renováveis. Oliveira e Ferreira (2018) comentam que a energia eólica, enquanto uma das formas de energias renováveis, tem se apresentado como um desafio para as políticas de desenvolvimento regional. No entanto, sabemos que as energias renováveis, como a eólica, são importantes no combate e controle das mudanças climáticas globais na medida em que contribuem para a redução das emissões de GEE, conforme discutiremos no tópico seguinte.

\section{Energias renováveis como alternativa de controle das mudanças climáticas}

\section{para as cidades brasileiras}

Dentre os ODS da ONU, as temáticas referentes à energia limpa e acessível referem-se ao ODS 07. De acordo com esse ODS e suas metas, dentre os compromissos das nações com a temática, destacam-se: a) assegurar o acesso confiável, sustentável, moderno e a preço acessível à energia para todas e todos; b) até 2030, aumentar substancialmente a participação de energias renováveis na matriz energética global; e c) até 2030, dobrar a taxa global de melhoria da eficiência energética (ONU, 2015). Num cenário em que as mudanças climáticas são uma grande ameaça às sociedades e aos ecossistemas, as energias renováveis, como eólica e solar, surgem como uma das alternativas centrais no controle das mudanças climáticas e na busca por cidades sustentáveis, pois além de proporcionar um menor impacto ambiental, seus recursos são, em sua maioria, inesgotáveis.

No Brasil, onde se tem observado um aumento do consumo e da produção de energia nas últimas décadas em relação às décadas de 1980 e 1990, podemos verificar um crescimento acentuado da utilização de energias renováveis, sobretudo eólica e solar (SILVA; SILVA; PESSOA, 2019). Em 2020, especificamente, verificamos o aumento de $5 \%$ e $57 \%$ do número de parques eólicos e de placas fotovoltaicas no Brasil em comparação a 2019, respectivamente (Tabela 1). 
Tabela 1 - Empreendimentos de energias eólica e solar por estados do Brasil (2020)

\begin{tabular}{|c|c|c|}
\hline Estados & $\begin{array}{l}\text { Quantidade de } \\
\text { empreendimentos de energia } \\
\text { eólica }\end{array}$ & $\begin{array}{l}\text { Quantidade de } \\
\text { empreendimentos de energia } \\
\text { solar }\end{array}$ \\
\hline Amapá & --- & 1 \\
\hline Amazonas & --- & 14 \\
\hline Bahia & 171 & 31 \\
\hline Ceará & 83 & 8 \\
\hline Espírito Santo & --- & 6 \\
\hline Maranhão & 16 & 3 \\
\hline Mato Grosso & --- & 1 \\
\hline Mato Grosso do Sul & $\begin{array}{c}-- \\
\end{array}$ & 3 \\
\hline Minas Gerais & 1 & 28 \\
\hline Paraíba & 15 & 4 \\
\hline Paraná & 1 & 4 \\
\hline Pará & --- & 3.708 \\
\hline Pernambuco & 35 & 4 \\
\hline Piauí & 60 & 20 \\
\hline Rio de Janeiro & 1 & 9 \\
\hline Rio Grande do Norte & 163 & 7 \\
\hline Rio Grande do Sul & 81 & 11 \\
\hline Roraima & $\begin{array}{l}--- \\
-\end{array}$ & 2 \\
\hline Santa Catarina & 16 & 5 \\
\hline São Paulo & 1 & 25 \\
\hline Sergipe & 1 & $\begin{array}{l}-- \\
\end{array}$ \\
\hline Tocantins & --- & 1 \\
\hline Total & 645 & 3.895 \\
\hline
\end{tabular}

Fonte: Teixeira et al. (2021, no prelo).

A energia eólica, produzida a partir da ação dos ventos, é considerada uma energia limpa, sustentável e com potencial de ser desenvolvida em todo litoral brasileiro, bem como em outros territórios, já que visualizamos um aumento de empreendimentos em cidades não litorâneas (Tabela 1). Cabe salientar, nessa direção, que os recursos renováveis não são tão limpos e sustentáveis, pois possuem impactos sobre as populações e os territórios onde são construídos, como apontam os estudos de Hofstaetter (2016) e Araújo et al. (2020) sobre os impactos socioambientais da energia eólica nos estados do RN e do CE, na região Nordeste do Brasil. De acordo com a Tabela 1, 
destacamos ainda a expansão da energia solar (aquela proveniente da luz e do calor do sol) no Brasil como uma das principais alternativas de energias renováveis. $O$ crescimento e uso da energia solar no Brasil vêm avançando e atraindo empresas.

Ademais, as energias renováveis vêm desempenhando, em nível global, um papel importante no contexto dos desafios que demandam energia elétrica, mas ao mesmo tempo atendimento às metas globais de mudanças climáticas. Jacobson et al. (2017) vêem nas energias eólica e solar, se implementadas em larga escala, um meio de atender completamente as necessidades mundiais de eletricidade. Outro princípio sobre o controle das mudanças climáticas está na ideia de que o armazenamento de eletricidade por meio de energias renováveis, como eólica e solar, é possível e viável a partir da utilização de baterias (DEHGHANI-SANIJ et al., 2019). Nesse ínterim, Geels et al. (2017) abordam que, no caso do setor elétrico, as transições aceleradas e profundas de baixo carbono, além de dependerem das inovações de energias renováveis, necessitam das inovações complementares, como o próprio armazenamento de energia elétrica (através de baterias, por exemplo).

Nesses termos, reforçamos que o crescimento na geração e produção de energias renováveis no Brasil, além de ser de suma importância para o alcance do ODS 07, é de fundamental relevância para outros ODS, tais como o 11 (relativo às cidades e comunidades sustentáveis) e o 13 (referente à ação contra a mudança global do clima). A integração desses ODS com o PNA é objeto de investigação do tópico em sequência.

\section{Adaptação climática no contexto das cidades brasileiras: pna e ods}

Para autores como Giddens (2010), as mudanças climáticas têm ganhado espaço no debate público, ocupando lugar de destaque nas agendas governamentais de vários países. Toda essa preocupação se dá em função dos avanços da ciência climática e dos relatórios do Painel Intergovernamental sobre Mudanças Climáticas (IPCC), órgão científico-político internacional criado em 1988 pelo Programa das Nações Unidas para o Meio Ambiente (PNUMA) e pela Organização Meteorológica Mundial (OMM), com vistas a produzir análises científicas de informações sobre as mudanças climáticas globais em 
curso, de modo a auxiliar os governos dos 195 países-membros no desenvolvimento de políticas públicas climáticas.

Os relatórios do IPCC apontam para cenários cada vez mais desafiadores, os quais incluem elevação do nível do mar, modificações na temperatura do ar e da precipitação pluviométrica, e ocorrência de eventos climáticos extremos. No centro dessa discussão, estão as cidades, que são consideradas grandes responsáveis pela intensificação das mudanças climáticas por meio das emissões de GEE (DODMAN, 2009; SATTERTHWAITE, 2008; WILBANKS et al., 2007); mas também são importantes no enfrentamento desse fenômeno a partir da elaboração e execução de estratégias de mitigação das emissões de GEE e de adaptação climática (AYLETT, 2014; LECK; ROBERTS, 2015; RYAN, 2015). Assim, reconhecemos a importância das cidades enquanto locais para abordar as questões do clima (HUITEMA; BOASSON; BEUNEN, 2018), reconhecendo vulnerabilidades, reduzindo riscos e criando estratégias resilientes.

Relativo à perspectiva da adaptação climática, os primeiros passos em sua direção foram por meio da criação do PNA, definido em 10 de maio de 2016, através da Portaria n. 2016, que tem como objetivo realizar uma gestão de riscos de eventos adversos provenientes das mudanças climáticas a fim de reduzir a vulnerabilidade nacional a essas mudanças (BRASIL, 2016). O PNA está organizado em 11 estratégias de adaptação para os setores e temas elencados como potencialmente vulneráveis às mudanças climáticas e, ao mesmo tempo, prioritários para o desenvolvimento do país (BRASIL, 2016). Face ao exposto, podemos associar a contribuição das estratégias setoriais do PNA para atingir as metas estabelecidas pelos ODS (Quadro 3), no sentido de incentivar os órgãos públicos no desenvolvimento de políticas e ações voltadas para o fomento da capacidade adaptativa de setores específicos, mas que pode refletir na resiliência dos demais.

\section{Quadro 3-Estratégias setoriais do PNA alinhados aos ODS}

\begin{tabular}{|l|l|}
\hline Estratégia setorial e temática & ODS \\
\hline $\begin{array}{l}\text { Agricultura - motivar e criar condições para } \\
\text { que o produtor rural possa estruturar e } \\
\text { manter sistemas de produção sustentáveis, }\end{array}$ & $\begin{array}{l}\text { Objetivo } 2 \text { - Acabar com a fome, } \\
\text { melhoria da nutrição e promover a }\end{array}$ \\
\hline
\end{tabular}


em sua diversidade de escala, tecnologia, natureza de mão de obra e direcionamento de mercado

Biodiversidade e Ecossistemas - integrar informações sobre mudança do clima às políticas e programas de conservação, recuperação e uso sustentável da biodiversidade

Cidades - identificar ações que contribuam diretamente para a redução da vulnerabilidade à mudança do clima e para o desenvolvimento de cidades resilientes

\section{Gestão de Risco e Desastres -}

promover ações visando desenvolver capacidades para a redução de riscos, preparação e resposta aos desastres no contexto da mudança do clima

Indústria e Mineração - introduzir a consideração do risco climático nas políticas públicas do setor e fomentar sua consideração nas decisões empresariais

Infraestrutura - aumentar a capacidade de resposta do setor de transportes frente aos eventos climáticos extremos

Povos e Populações Vulneráveis - apoiar na contextualização e na identificação de grupos populacionais vulneráveis à mudança do clima

Recursos Hídricos - planejar a operação dos Recursos Hídricos à variabilidade e mudanças climáticas

Saúde - monitorar e comunicar clima e saúde para ampliação do conhecimento técnico-científico, e subsídio à análise de situação de saúde e à tomada de decisão agricultura sustentável

Objetivo 15 - Proteger, recuperar e promover o uso sustentável dos ecossistemas terrestres, gerir de forma sustentável as florestas, combater a desertificação, deter e reverter a degradação da terra e deter a perda de biodiversidade

Objetivo 11 - Tornar as cidades e os assentamentos humanos inclusivos, seguros, resilientes e sustentáveis

Objetivo 13 - Tomar medidas urgentes para combater a mudança do clima e seus impactos

Objetivo 9 - Construir infraestruturas resilientes, promover a industrialização inclusiva e sustentável e fomentar a inovação

Objetivo 9 - Construir infraestruturas resilientes, promover a industrialização inclusiva e sustentável e fomentar a inovação

Objetivo 1 - Acabar com a pobreza em todas as suas formas, em todos os lugares

Objetivo 10 - Reduzir a desigualdade dentro dos países e entre eles

Objetivo 6 - Assegurar a disponibilidade e gestão sustentável da água e saneamento para todos

Objetivo 3 - Assegurar uma vida saudável e promover o bem-estar para todos, em todas as idades 


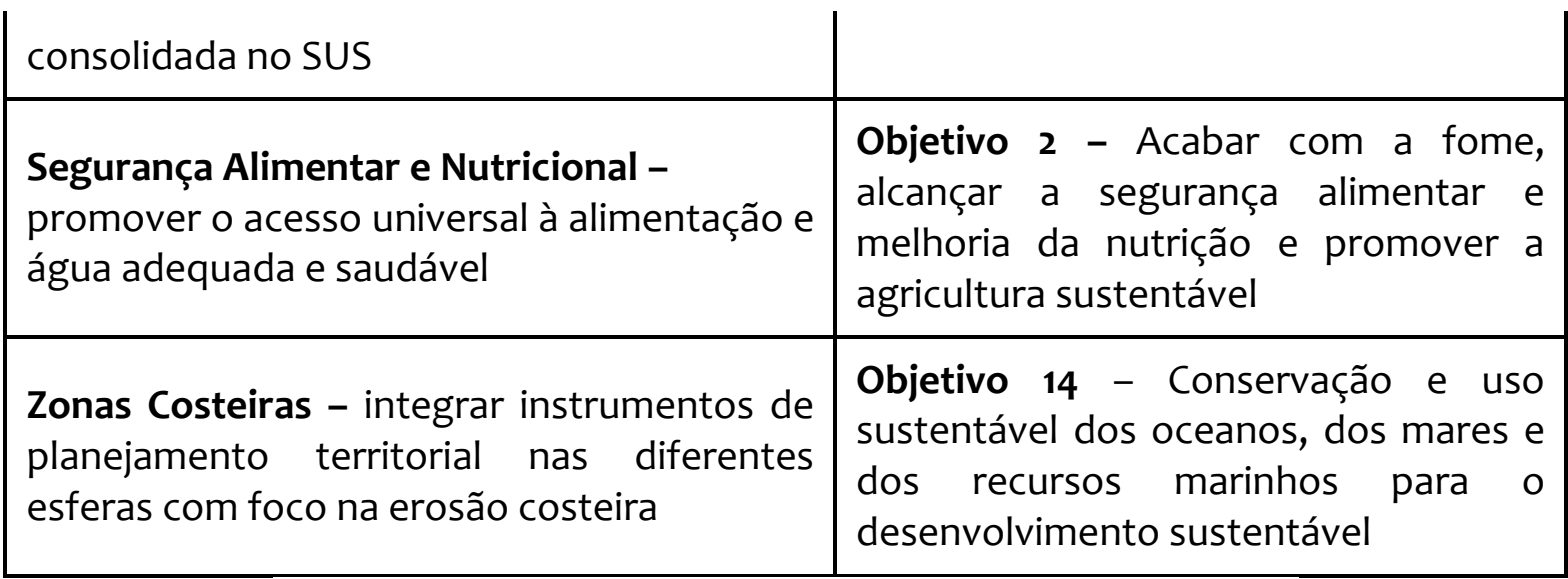

Fonte: Elaboração própria a partir da ONU (2015) e Brasil (2016).

Nota: Um quadro mais sintético dessa natureza compõe o estudo realizado por Teixeira et al. (2021, no prelo).

O desenvolvimento sustentável e as estratégias de adaptação às mudanças climáticas são indissociáveis, tendo em vista que o desenvolvimento sustentável agrega as esferas econômicas, ecológicas e humanas, que são condições essenciais para a manutenção do equilíbrio climático mundial. Nessa ótica, é importante destacar que, na prática, o PNA e tantas outras iniciativas de mudanças climáticas do Brasil não têm efetividade no contexto das cidades, pois estas contam com gestões públicas que, quando definem objetivos, princípios e/ou diretrizes voltados para a questão das mudanças climáticas locais, não os estabelecem levando em consideração às particularidades de suas realidades: o que acontece, na maioria das vezes, é a replicação de estratégias já existentes para suas respectivas realidades.

Com isso, as cidades brasileiras apresentam desafios quanto à capacidade de lidar com os efeitos negativos das mudanças climáticas. Esse aspecto pode ser corroborado no estudo de Di Giulio et al. (2019b), que ao analisarem seis grandes cidades brasileiras com diferentes níveis de vulnerabilidade sócio-climática (Curitiba, Porto Alegre, São Paulo, Vitória, Manaus e Natal), concluem que um dos grandes e complexos desafios a serem enfrentados pelas cidades brasileiras é a efetivação de capacidade adaptativa climática enquanto estratégia essencial para a construção de um mundo sustentável, combinando a equidade social, o crescimento econômico e o uso dos recursos naturais. Autores como Teixeira e Pessoa (2020) e Teixeira, Pessoa e Di Giulio (2020), ao estudarem 
respectivamente as cidades de Curitiba e Natal, também apontam esse desafio. No próximo tópico, concluímos essas considerações, ressaltando que a inserção da adaptação climática, enquanto um mecanismo de incentivo à construção de uma sociedade sustentável, ainda não se configura em uma agenda estratégica para as cidades brasileiras.

\section{Conclusões}

Os desafios para a inserção de estratégias de capacidade adaptativa às mudanças climáticas alinhadas à Agenda 2030 são grandes para as cidades em todo mundo. No caso do Brasil, devido às assimetrias urbanas e sociais e ao desenvolvimento histórico, esses desafios exigem mais comprometimento de todos os segmentos da sociedade, inclusive dos governos em todos os seus níveis de poder.

É perceptível que mudanças climáticas estão em curso. Um dos mais recentes relatórios do IPCC, divulgado em outubro de 2018, ratifica que estamos vivendo essas mudanças e que decorrem dos estilos de desenvolvimento que têm nas cidades seus espaços naturais de reprodução - a cultura do consumo e do descarte (IPCC, 2018). Mudar esses estilos e que se voltem para a emergência de modelos sustentáveis com justiça ambiental e menor vulnerabilidade é o maior desafio para as cidades, sejam brasileiras ou não (MADEIROS; GRIGIO; PESSOA, 2018)

Para isso, efetivar a Agenda 2030 é essencial e provocativo, pois tradicionalmente no Brasil, apesar dos avanços da Política Nacional do Meio Ambiente (PNMA), definida pela Lei $n^{\circ} 6.938$, de 31 de agosto de 1981, e de todo o conjunto de políticas públicas ambientais cumulativas nessas últimas décadas, a questão ambiental segue marginal nas agendas, especialmente, dos governos municipais.

Precisamos pontuar ainda que vivemos um cenário bastante preocupante no contexto nacional, quando se tem um sistema de governo em que as agendas urbana e ambiental não se configuram em estratégias prioritárias a partir de janeiro de 2019. Nessa perspectiva, todos os avanços na gestão urbana e ambiental brasileira vêm sofrendo retrocessos significativos e que podem comprometer a sustentabilidade das cidades no 
futuro próximo, caso seja ignorada a Agenda 2030 nos âmbitos federal, estadual e municipal.

Essa marginalidade e secundarização nas agendas das cidades brasileiras ficam evidentes quando observamos que poucas cidades têm planos de mitigação e de adaptação climática, ao lado de um déficit de sistemas de monitoramento, análise e avaliação de áreas de riscos. Além disso, muitas cidades, sobretudo na região Nordeste, são produtoras de energias renováveis; no entanto, não consomem essa energia produzida (HOFSTAETTER; PESSOA, 2015a; HOFSTAETTER; PESSOA, 2015b). Enfim, muitos são os desafios das cidades na perspectiva da efetivação de suas capacidades adaptativas às mudanças climáticas, mas seguimos firmes de que o caminho da sustentabilidade é o mais viável para o futuro das cidades do Brasil e do mundo.

\section{Referências}

ALMEIDA, F. Gomes de. O ordenamento territorial e a geografia física no processo de gestão ambiental. In: SANTOS, Milton et al. (orgs.). Território, território: ensaios sobre o ordenamento territorial. Rio de Janeiro: Lamparina, 2007.

APOLLARO, Camila; ALVIM, Angélica. Estratégias e desafios do planejamento urbano para a adaptação de cidades frente à mudança climática. Revista Meio Ambiente e

Sustentabilidade, v. 13, n. 6, 2017.

\section{RAÚJO, Ana Célia Baía. Desenvolvimento urbano-regional e cidades sustentáveis no} contexto das regiões metropolitanas nordestinas. 2019. 166f. Dissertação (Mestrado em Estudos Urbanos e Regionais) - Centro de Ciências Humanas, Letras e Artes, Universidade Federal do Rio Grande do Norte, Natal, 2019.

ARAÚJO, Júlio César Holanda et al. Sustainability challenges of wind power deployment in Coastal Ceará State, Brazil. Sustainability, v. 12, n. 14, p. 5562, 2020.

AYLETT, Alexander. Progress and challenges in the urban governance of climate change: results of a global survey. Cambridge, MA: MIT, 2014.

BRASIL. Ministério do Meio Ambiente. Lei n 6.938, de 31 de agosto de 1981. Dispõe sobre a Política Nacional do Meio Ambiente, seus fins e mecanismos de formulação e aplicação, e dá outras providências. Brasília: Presidência da República, 1981. Disponível em:

http://www.planalto.gov.br/ccivil_03/leis/L6938.htm. Acesso em: 07 de set. de 2020. 
BRASIL. Ministério da Integração Regional. Para pensar uma política nacional de ordenamento territorial: anais da oficina sobre a política nacional de ordenamento territorial, Brasília, 13-14 de novembro de 2003/Ministério da Integração Nacional, Secretaria de Políticas de Desenvolvimento Regional (SDR). Brasília: O Ministério, 2005. 78 p. Disponível em:

https://www.mdr.gov.br/images/stories/ArquivosSNPU/Biblioteca/publicacoes/ordename nto territorial.pdf. Acesso em: 30 abr. 2020.

BRASIL. Ministério do Meio Ambiente. Plano nacional de adaptação à mudança do clima: sumário executivo. Brasília, DF: O Ministério, 2016. Disponível em:

https://www.mma.gov.br/images/arquivo/80182/LIVRO_PNA_Resumo\%20Executivo_.pdf. Acesso em: 30 abr. 2020.

DEHGHANI-SANIJ, Alireza et al. Study of energy storage systems and environmental challenges of batteries. Renewable and Sustainable Energy Reviews, v. 104, p. 192-208, 2019.

DI GIULIO, Gabriela Marques et al. Eventos extremos, mudanças climáticas e adaptação no estado de São Paulo. Ambiente \& Sociedade, São Paulo, v. 22, p. 3-20, 2019 a.

DI GIULIO, Gabriela Marques et al. Bridging the gap between will and action on climate change adaptation in large cities in Brazil. Regional Environmental Change, v. 19, n. 8, p. 2491-2502, 2019b.

DODMAN, David. Blaming cities for climate change?:an analysis of urban greenhouse gas emissions inventories. Environment and urbanization, v. 21, n. 1, p. 185-201, 2009.

EAKIN, Hallie, LEMOS, Maria Carmen; NELSON, Donald. Differentiating capacities as a means to sustainable climate change adaptation. Global Environmental Change, v. 27, p. $1-8,2014$.

ESPÍNDOLA, Isabela Battistello; RIBEIRO, Wagner Costa. Cidades e mudanças climáticas: desafios para os planos diretores municipais brasileiros. Cadernos Metrópole, v. 22, n. 48, p. 365-396, 2020.

GEELS, Frank W. et al.Sociotechnical transitions for deep decarbonization. Science, v. 357, n. 6357, p. 1242-1244, 2017.

GIDDENS, Anthony. A política da mudança climática. Rio de Janeiro: Zahar. 2010. 314 p.

HOFSTAETTER, Moema; PESSOA, Zoraide. Impactos Socioambientais e Regionais da Energia Eólica no Rio Grande do Norte. In: ENCONTRO NACIONAL DA ANPPAS, 7., 2015, Brasília. Anais [...]. Brasília: UNB, 2015a, p. 1-15. 
HOFSTAETTER, Moema; PESSOA, Zoraide. Energia eólica: um novo debate, entre defesas e contradições. In: JORNADA INTERNACIONAL DE POLÍTICAS PÚBLICAS, 7., 2015, São Luís. Anais [...]. São Luís: UFMA, 2015b. , p. 1-11.

HOFSTAETTER, Moema. Energia eólica: entre ventos, impactos e vulnerabilidades socioambientais no Rio Grande do Norte. 2016. 176 f. Dissertação (Mestrado em Estudos Urbanos e Regionais) - Universidade Federal do Rio Grande do Norte, Natal, 2016.

HUITEMA, Dave; BOASSON, Elin Lerum; BEUNEN, Raoul. Entrepreneurship in climate governance at the local and regional levels: concepts, methods, patterns, and effects. Regional Environmental Change, v. 18, p. 1247-1257, 2018.

IBGE - INSTITUTO BRASILEIRO DE GEOGRAFIA E ESTATÍSTICA. Plataforma digital dos objetivos de desenvolvimento sustentável (plataforma ODS). Rio de Janeiro: IBGE, 2018.Disponível em: https://odsbrasil.gov.br/. Acesso em: 230ut. 2019.

IPCC - PAINEL INTERGOVERNAMENTAL SOBRE MUDANÇAS CLIMÁTICAS. Climate change 2007: impacts, adaptation and vulnerability: contribution of working group II to the fourth assessment report of the intergovernmental panel on climate change,2007. UNITED KINGDOM: CROWN, 2007. Disponível em: https://www.ipcc.ch/site/assets/uploads/2018/03/ar4_wg2_full_report.pdf. Acesso em: 28 abr. 2020.

IPCC - PAINEL INTERGOVERNAMENTAL SOBRE MUDANÇAS CLIMÁTICAS. Aquecimento global de $1,5^{\circ} \mathrm{C}$ : sumário para formuladores de políticas. [S.I.]: IPCC, 2018. Disponível em: https://www.ipcc.ch/site/assets/uploads/2019/07/SPM-Portuguese-version.pdf. Acesso em: 01 abr. 2020.

JACOBSON, Mark Zachary et al. 100\% clean and renewable wind, water, and sunlight allsector energy roadmaps for 139 countries of the world. Joule, v. 1, n. 1, p. 108-121, 2017.

LECK, Hayley; ROBERTS, Debra. What lies beneath: understanding the invisible aspects of municipal climate change governance. Current Opinion in Environmental Sustainability, v. 13, p. 61-67, 2015.

MADEIROS, Heleriany; GRIGIO, Alfredo; PESSOA, Zoraide. Desigualdades e justiça ambiental: um desafio na construção de uma cidade resiliente. GOT, Porto, n. 13, p. 247265, 2018.

MORAES, A. Carlos R. Ordenamento territorial: uma conceituação para o planejamento estratégico. In: MORAES, A. Carlos R. (org.).Meio ambiente e ciências humanas. 4. ed. São Paulo: Annablume, 2005. p. 139-149. 
OLIVEIRA, Herbert Emammanuel Lima de; FERREIRA, José Gomes. Energia eólica e desenvolvimento regional no semiárido do Rio Grande do Norte: uma proposta de análise. In: CONGRESSO NACIONAL SOBRE A DIVERSIDADE DO SEMIÁRIDO, 1.,2018. Natal. Anais [...]. Campina Grande: Realize Eventos e Editora, 2018, p. 1-10.

ONU - ORGANIZAÇÃO DAS NAÇÕES UNIDAS. Transformando nosso mundo: a agenda 2030 para o desenvolvimento sustentável. Nova York: ONU, 2015. Disponível em: https://nacoesunidas.org/wp-content/uploads/2015/10/agenda2030-pt-br.pdf. Acesso em: 03 de jul. de 2020.

PESSOA, Zoraide Souza. A metrópole periférica: identidade e vulnerabilidade socioambiental na região metropolitana de Natal-RN/Brasil. 2012. 276 f. Tese (Doutorado em Ambiente e Sociedade) - Universidade Estadual de Campinas, Campinas, 2012.

RÜCKERT, Aldomar A. A política nacional de ordenamento territorial, Brasil: uma política territorial contemporânea em construção. Scripta Nova: revista electrónica de geografía y ciências sociales, v. 11, 2007.

RYAN, Daniel. From commitment to action: a literature review on climate policy implementation at city level. Climatic Change, v. 131, n. 4, p. 519-529, 2015.

SATHLER, Douglas; PAIVA, Júlio César; BAPTISTA, Sandra. Cidades e mudanças climáticas: planejamento urbano e governança ambiental nas sedes das principais regiões metropolitanas e regiões integradas de desenvolvimento. Caderno de Geografia, Belo Horizonte, v. 29, n. 56, p. 262-262, 2019.

SATTERTHWAITE, David. Climate change and urbanization: effects and implications for urban governance. In: UNITED NATIONS EXPERT GROUP MEETING ON POPULATION DISTRIBUTION, URBANIZATION, INTERNAL MIGRATION AND DEVELOPMENT. New York: Desa, 2008. p. 21-23.

SILVA, Alice Leonora de Sousa; SILVA, Loren Cassiane Souza; PESSOA, Zoraide Souza. Energia eólica e solar: produção no Brasil e o impacto socioambiental nos municípios do RIO GRANDE DO NORTE (RN). In: ENCONTRO NACIONAL DE ENSINO E PESQUISA DO CAMPO DE PÚBLICAS (ENEPCP), 3., 2019, Natal. Anais [...]. Brasília: ANEPCP, 2019, p. 3036-3049.

TEIXEIRA, Rylanneive; PESSOA, Zoraide. Mudanças climáticas, experimentação de políticas públicas e capacidade adaptativa na cidade de Curitiba/PR-Brasil. Revista InterLegere, v. 3, n. 27, p. 1-28, 2020. 
TEIXEIRA, Rylanneive; PESSOA, Zoraide; DI GIULIO, Gabriela. Mudanças climáticas e capacidade adaptativa no contexto da cidade do Natal/RN, Brasil. Revista Geotemas, v. 10, n. 1, p. 95-115, 2020.

TEIXEIRA, Rylanneive et al. Mudanças climáticas, capacidade adaptativa e sustentabilidade: reflexões a partir das cidades da região semiárida brasileira. Revista Geotemas, v. 11, n. 1, 2021. No prelo.

WILBANKS, Thomas J. et al. Industry, settlement and society. In: CLIMATE CHANGE 2007: impacts, adaptation and vulnerability, contribution of working group II to the fourth assessment report of the intergovernmental panel on climate change. Cambridge University Press: IPCC, 2007. p. 357-390. Disponível em: https://www.ipcc.ch/site/assets/uploads/2018/02/ar4-wg2-chapter7-1.pdf. Acesso em: 30 abr. 2020. 